EPJ manuscript No.

(will be inserted by the editor)

\title{
How does flow in a pipe become turbulent?
}

\author{
Bruno Eckhardt ${ }^{1}$ and Tobias M. Schneider ${ }^{1}$ \\ Fachbereich Physik, Philipps-Universität Marburg, 35043 Marburg \\ Received: date / Revised version: date
}

\begin{abstract}
The transition to turbulence in pipe flow does not follow the scenario familiar from RayleighBenard or Taylor-Couette flow since the laminar profile is stable against infinitesimal perturbations for all Reynolds numbers. Moreover, even when the flow speed is high enough and the perturbation sufficiently strong such that turbulent flow is established, it can return to the laminar state without any indication of the imminent decay. In this parameter range, the lifetimes of perturbations show a sensitive dependence on initial conditions and an exponential distribution. The turbulence seems to be supported by threedimensional travelling waves which appear transiently in the flow field. The boundary between laminar and turbulent dynamics is formed by the stable manifold of an invariant chaotic state. We will also discuss the relation between observations in short, periodically continued domains, and the dynamics in fully extended puffs.
\end{abstract}

PACS. 47.27.Cn Transition to turbulence - 47.27.nf Flows in pipes and nozzles - 47.10.Fg Dynamical systems methods

\section{Introduction}

Dynamical system theory and nonlinear dynamics has figured prominently in understanding the transition to turbulence in systems with linear instabilities of the laminar profile 11. The various bifurcations and transitions in fluids heated from below (Rayleigh-Benard) and in the flow between independently rotating cylinders (TaylorCouette) have been studied in considerable details and have helped to advance both our understanding of the various transition scenarios and of the properties of dy' namical systems in general. Pressure driven flow in a pipe , or the flow between moving parallel plates (plane Couette flow) behave differently and are less well understood: the linear stability of the laminar profile prevents the immediate application of the usual bifurcation and transition scenarios 22345. So how does the turbulence observed in experiments come about?

Evidence that has emerged in recent years suggests that dynamical systems also provide a role model for the transition in linearly stable situations 6 6/57. The application of these ideas to the typically fairly high-dimensional situation of pipe flow also raises interesting questions about dynamical systems, so that one can again expect a fruitful interplay between dynamical systems theory and the fluid dynamics of the transition to turbulence. The focus of this contribution will be to survey recent progress in our understanding of pipe flow. We will go beyond previous studies 8415. by including steps towards a characterization of the spatial variability of the system.
The main features of turbulence transition in a pipe were documented long ago by Osborne Reynolds [910. He noticed the possibility to delay the transition by suppressing perturbations in the inflow region, the intermittent character of the transition, and the presence of vortices in the turbulent regions. He noticed that the flow can be characterized by a dimensionless combination of mean downstream velocity $U$, radius of the pipe $R$ and kinematic viscosity $\nu$, nowadays known as the Reynolds number $R e=2 U R / \nu$. The intermittent character of the transition and the absence of a linear instability of the laminar profile make the determination of a "critical Reynolds number' above which turbulence can be observed a tricky business, as attested by the huge variability of critical numbers that can be found in the literature. Reynolds arrives at about 1800, Prandtl [11] speculates that it should be above 1000, Mullin and Darbyshire [12] find long lived turbulent trajectories above about 1650 , and most reference books and textbooks quote numbers in the range of 2000 to 3000 . The operational definition would be that the critical Reynolds number is defined such that sufficiently strong perturbations can induce turbulence if the critical value is exceeded. But even with that definition there remain problems connected with the transience of the turbulent state, as discussed in section 5 .

An interesting feature of turbulence in pipe flow is that below Reynolds numbers of about 2700, it does not extend throughout the pipe but remains localized in turbulent puffs of about $60 R$ length, 13]14. While some progress has been made in describing localized structures anf frontal dynamics in various pattern forming systems 


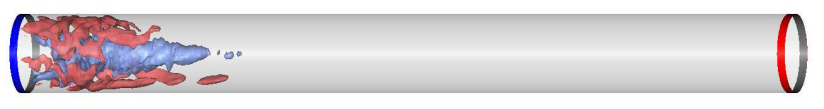

Fig. 1. Three-dimensional flow inside a puff at $R e=1825$ moving from left to right. The snapshots are separated by time intervals of $t=40 R / u_{c l}$ and show isosurfaces of the downstream velocity relative to the laminar profile at levels $\pm 0.1 u_{c l}$ (negative in blue and positive in red). The plots are compressed by a factor of 5 in the axial direction to show the whole computational domain of $L=100 R$. Three more frames not included for size reasons.

[15], the case of localized turbulence is more complicated because of the dynamic nature of the localized state [16. Some progress has recently been made for the case of localized turbulent stripes in Taylor-Couette and plane Couette flow, 17/18.

For our presentation below we will draw on some experimental observations, and on numerical simulations of the full Navier-Stokes equation in domains of length 100R (for the spatially extended puffs) and 10R (for the statistical studies in smaller domains), where $R$ is the radius. The numerical code has been verified by reproducing the spectrum of the linearized equation, and the turbulent properties at somewhat higher Reynolds. The coherent structures have also been confirmed by independent studies.

The outline of the paper is as follows. In section 2 we discuss the dynamics of a localized turbulent puff and present evidence that the interior dynamics can be captured by studies in smaller domains. In section 3 we discuss coherent states and their bifurcations in domains of length 10R. In secion 4 we present lifetime studies in the small domain and relate them to experiment and other investigations. In section 5 we discuss the boundary between the laminar and turbulent regions in state space and the evidence for the presence of an edge of chaos as a generalization to the usual basin boundaries. In section 6 we summarize and give a brief outlook how this relates to studies in other systems.

\section{Puffs}

In the Reynolds number range up to about 2700 , the transition to turbulence in pipe flow shows a striking spatial localization 1314: the turbulence is concentrated in regions of about 60R length, which move uniformly through the pipe [13|14|19|20|21/22|23]. In Fig. 1 we present four snapshots of a puff and in Fig. 2 the axial position of its center, demonstrating the very uniform speed with which it moves downstream. The position of the puff is defined via the center of the turbulent in-plane kinetic energy distribution. This criterion is very robust as compared to one based on a jump in the downstream centerline velocity (see [24), and gives almost noise-free position information and very accurate translation velocities, as evident from Fig. 2, where no smoothing or linear interpolation was applied.

The axial extension of the structure remains fairly constant and its traveling velocity decreases form $0.965 U$ of

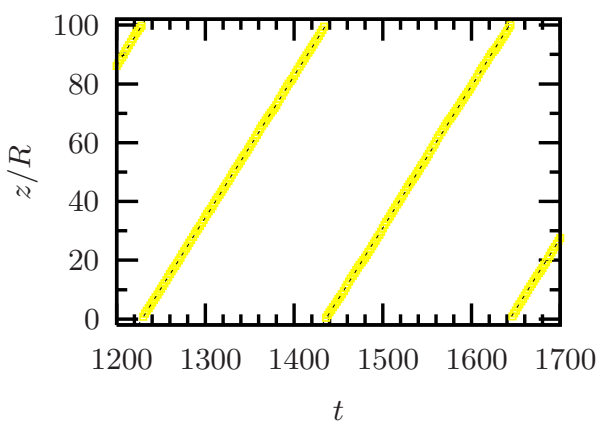

Fig. 2. Uniform motion of the puff along the pipe. The yellow line is the position of the center of turbulent energy in the in-plane velocity components, the dashed line a linear approximation to it. Shown are two passages through the pipe (which in the numerical simulations is periodically continued along the axis). The Reynolds number is $R e=1800$.

the mean downstream velocity at $\mathrm{Re}=1800$ to $0.94 \mathrm{U}$ at $\operatorname{Re}=1900$. Thus, the turbulent patch is slightly slower than the bulk velocity of the fluid. Consequently, when viewed from a frame of reference comoving with the puff, there is a net flow of fluid from the trailing to the leading edge of the puff.

The velocity field inside the puff can be divided up into three regions, a fairly homogeneous turbulent interior bracketed by the upstream and downstream front regions. In order to highlight the different characteristics of the velocity fields in these domains, we apply the correlation function measures introduced in 26] to extract coherent structures. Since in the situation of a localized puff the system is not only inhomogeneous in wall-normal but also in axial direction we only take out the mean velocity as obtained from averages over the azimuthal direction, the only homogeneous direction left. Thus, the correlation functions are evaluated for the local deviations from the downstream speed as calculated along a ring at radius $r=0.81$ :

$$
\tilde{u}_{z}(\phi, z)=u_{z}(r=0.81, \phi, z)-\left\langle u_{z}(r=0.81, \phi, z)\right\rangle_{\phi}
$$

and

$$
C(\phi, z)=\left\langle\tilde{u}_{z}(\psi+\phi, z) \tilde{u}_{z}(\psi, z)\right\rangle_{\psi}
$$

Snapshots of this correlation function in Fig. 3 clearly show the three different regions. Intriguingly, the upstream and downstream regions are very often dominated by pronounced coherent structures. On the upstream side, structures with three and four streaks dominate. Thus, the center panel of Fig. 3 shows a typical flow configuration. On the downstream side, the structures are somewhat shorter and occur not quite as often as on the upstream side. The dynamics of these coherent structures and their significance for the transition to turbulence was studied extensively by Cas van Doorne in his PhD thesis, 27. The central region is spatially and temporally more disordered.

The disorder in the interior domain is also reflected in rapidly decaying spatial auto-correlation functions of the three velocity components, see Fig. 4. The downstream 


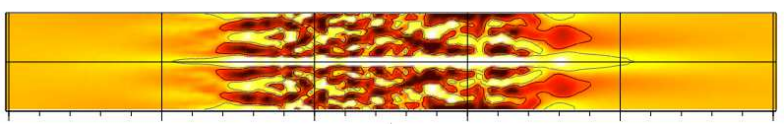

Fig. 3. Coherent structures in a turbulent puff at three different times for $R e=1800$. The abscissa runs from $z=10 R$ to $z=60 R$ (half of the computational domain of $L=100 R$ ) and is translated so that the center of the turbulent intensity is kept at $z=L / 3$. The ordinate shows the angle $\phi$ from $-\pi$ to $\pi$ with the 0 level indicated by the horizontal line. White shading indicates positive values, dark shading negative values of the correlation function $C(\phi, z)$. Note the rather disordered interior and the elongated coherent structures at the upstream and downstream edges of the puff. Two more frames not included because of size problems.

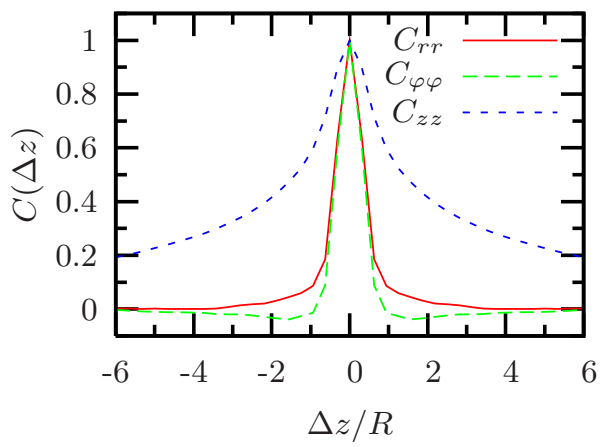

Fig. 4. Autocorrelation functions for the downstream $\left(C_{z z}\right)$, azimuthal $\left(C_{\varphi \varphi}\right)$ and radial $\left(C_{r r}\right)$ velocities along the axis evaluated in the comoving frame of reference. The correlations are normalized to one for vanishing axial shift $\Delta z$. They are based on the deviation from the time and area averaged mean profiles, resolved along the axis. The correlation functions are averaged over $3 / 4$ of the computational domain. They include the entire puff, and thus also the elongated coherent structures at the up- and downstream boundaries. The time average is based on about 1000 independent velocity fields. The presented curves are for a radial position of $r=0.73$, but not much variation with $r$ was observed.

component shows the longest axial correlation length due to the presence of streaks. As measured by the level it reaches $1 / \mathrm{e}$, it extends over a width of about $2 R$. The correlation functions for the other components drop to $1 /$ e of their maximal value over distances of less than one pipe radius. The short axial correlation length suggests that useful information about the interior dynamics of the flow can be obtained by studying relatively short domains. Specifically, for a length of $10 R$ the axial correlations in the downstream fluid are down to 30 per cent, but the computational advantages are enourmous and allow for detailed studies of deterministic [28] and statistical analyses [26].

\section{Local dynamics in short domains}

The correlation functions of the previous section suggest that it should be possible to study the dynamics in short domains, thereby eliminiating much of the large-scale spatial dynamics. Nevertheless, the state space of the system remains fairly high-dimensional, and easily reaches $\mathcal{O}\left(10^{5}\right)$ active dynamical degrees of freedom after the elimination of boundary conditions and incompressibility.

The coexistence of laminar and turbulent dynamics for the same parameter values but different initial conditions in this system suggests that the transition might be associated with a subcritical bifurcation (compare [29]). For increasing Reynolds number, the usual scenario would then imply the appearance of new states in a saddle-node bifurcation, followed by the destabilization of the laminar profile in a collision with the saddle (as realized, for instance, in TC flow in the narrow gap limit, 30]).

In the case of pipe flow new persistent flow structures besides the laminar profile have indeed been identified 2831. They are of the type of full three-dimensional travelling waves, see Fig. 5. Three-dimensionality is required, since velocity fields with translational symmetry in the downstream direction decay and cannot sustain any non-trivial flow state. The first structures identified were highly symmetric, and contained several pairs of vortices. Among these, the structure with the lowest critical Reynolds number is the threefold set of vortices, with a bifurcation near $R e=1250$. It is followed by the bifurcation of the twofold state at Reynolds number $R e=1350$, and more at higher Reynolds number. Interestingly, the friction factors are sometimes higher than the turbulent ones, but they tend to approach the laminar values for higher Reynolds number, Fig. 6] Less symmetric structures may also be identified, and some of them extend to lower Reynolds numbers 32 .

All these states actually belong to continuous families, since they tolerate some stretching and compression for Reynolds number above their point of bifurcation, [287. However, their optimal wavelengths at the bifurcation of $2.5 R$ and $4.2 R$ for three and two pairs of vortices, respectively, 28, can fit well into the short domains studied in the next section.

The bifurcation scenario in pipe flow, however, differs from the usual one in that both states that appear in the bifurcation are immediately unstable [7]: it is like a saddle node bifurcation in an unstable subspace. This has two consequences. As a first one we note that the absence of stable patterns and the hyperbolic dynamics on the saddle naturally explain the chaotic dynamics. Nevertheless, the coherent structures just discussed can show up transiently in the dynamics [25, where they can be detected by application of the indicator (2). Studies on time series then show that about 20 per cent of the time the flow is dominated by the presence of these structures [26].

Another consequence of this deviation from the standard saddle-node transition scenario is the fact that the stable manifold of the saddle is not of co-dimension one and therefore does not subdivide state space into the domains of attraction of the laminar and the turbulent dynamics. Moreover, the saddle does not collide with the laminar profile for any finite Reynolds number: the laminar profile remains stable against infinitesimal perturba- 


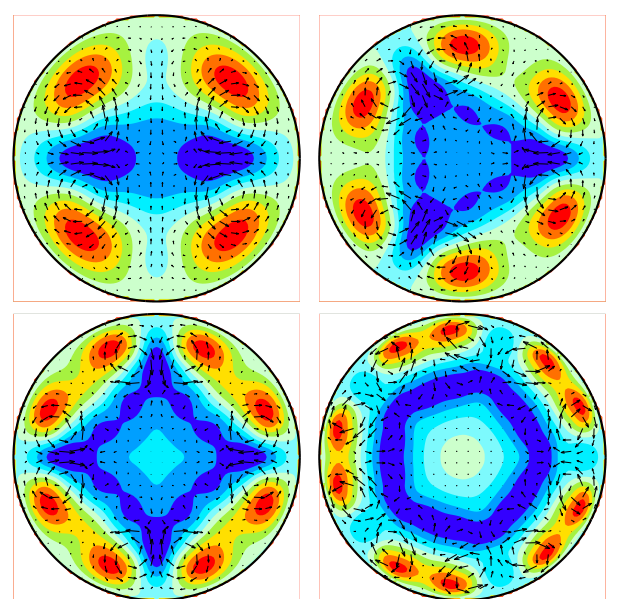

Fig. 5. Coherent states for pipe flow. Shown are velocity profiles averaged in the downstream direction. The deviation from the laminar Hagen-Poseuille profile is indicated by colors. Negative velocities are shown in blue, positive areas in red. In-plane velocity components are indicated by vectors. The regular arrangement of high- and low-speed streaks reflects different discrete rotational symmetry classes.

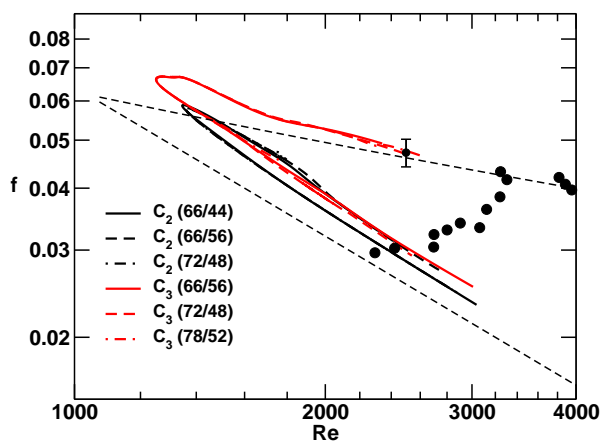

Fig. 6. Coherent structures in a friction factor vs. Reynolds number plane for pipe flow. $C_{2}$ and $C_{3}$ indicate states with two and three vortex pairs, respectively, and the numbers in parenthesis indicate different resolutions (see [28]). The dots indicate observations on a turbulent flow, and the upper and lower dashed lines are extrapolations of the turbulent and laminar behaviour, respectively.

tions for all Reynolds numbers 33 . This raises interesting questions about the boundary between laminar and turbulent dynamics to which we turn in section 5, and it opens up the possibility that the turbulent dynamics is not persistent but transient, a feature we analyze in the next section.

\section{Lifetimes}

In the usual subcritical bifurcation scenario the stable node forms the basis for an attracting subset in state space, where chaos can set in as a result of further bifurcations. However, in the absence of a stable object, i.e. if the bifurcation happens within a subspace that is al- ready unstable with respect to perpendicular deviations, the persistence of the dynamics in the turbulent region has to be investigated separately. Indeed, studies by Brosa 34 first suggested the intriguing possibility that turbulence might be transient. As a first step in this direction we study lifetimes of a sample of initial conditions in the turbulent region. Numerical 35/36/37 and experimental studies 19202112236 show that in this region the lifetimes are exponentially distributed. Asymptotically, for long times, the probability to find a turbulent state after a time $t$ is given by

$$
P(t) \propto e^{-\left(t-t_{0}\right) / \tau} \quad \text { for } \quad t>t_{0},
$$

with a characteristic decay time $\tau$. The exponential distribution for $P(t)$ implies that the probability density $p(t)=$ $d \ln P(t) / d t$ to decay at a time $t$ is constant: the flow has no memory and decays at unpredictable moments in time. This constant decay probability is strong evidence for the formation of a chaotic saddle that supports transient turbulent dynamics. The characteristic decay time $\tau$ increases rapidly with Reynolds number, but it does not seem to diverge at any finite $R e$. This is confirmed in experiments on long pipes and in numerical simulations as shown in Fig. 7 [36. Other experimental studies on shorter pipes $19 \sqrt[2012122]{ }$ and simulations for five Reynolds numbers [38 have been interpreted using a variation $\tau(R e) \sim a /\left(R e_{c}-R e\right)$ : however, the extracted parameter $a$ and the critical Reynolds number $R e_{c}$ vary over a wide range, leaving questions whether the asymptotic regime has indeed been reached. Moreover, a reanalysis of the data 39 shows that the numerical simulations are fairly close to the experimental results from Hof et al [36. The comparison between the data suggests the interesting possibility that a global bifurcation near Reynolds numbers of about 1850 (see also 40) might result in a quantitative change of the scaling function parameters. Perhaps previously separated parts of the turbulence supporting scaffold become connected to raise the lifetimes, but without actually turning the turbulent saddle into an attractor.

The observation of transient turbulence complicates the identification of a critical Reynolds number for the transition to turbulence: ideally, this would be the Reynolds number where the flow becomes persistently turbulence. The absence of a divergence in the lifetimes rules this definition out. Alternatively, one might require that more than half of all initial conditions remain turbulent up to some time: then the critical Reynolds number depends on the probability and the observation time. Fortunately, these dependences are fairly weak on account of the rapid increase with Reynolds number: with the data from [36], the characteristic time $\tau$ (measured in units of $2 R / U$ ) reaches 1000 for a Reynolds number of 1924, and 2000 already for 1944 .

\section{Edge of chaos}

The coexistence of a chaotic saddle carrying the turbulent dynamics and the linearly stable fixed point surounded by 

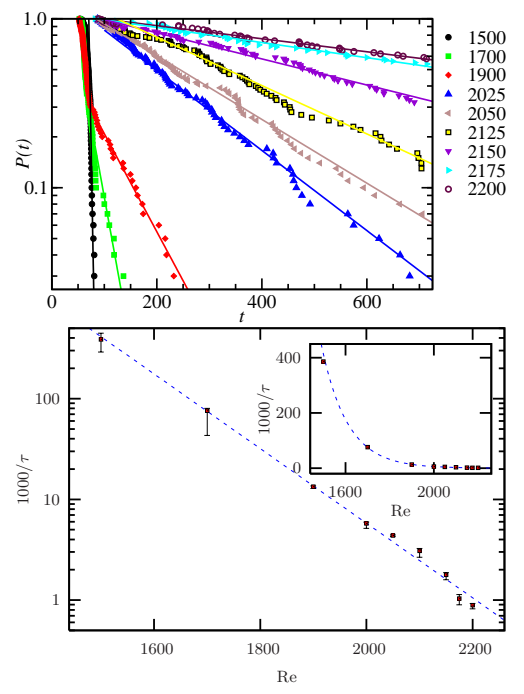

Fig. 7. Lifetime distributions for pipe flow, and variation of mean lifetime with $R e$ for a pipe segment of length $10 R$. Top panel: Probability to still be turbulent after some time $t$ for several Reynolds numbers in a semi-logarithmic plot. The straight lines indicate exponential fits to the tails of the distributions. Bottom panel: Inverse characteristic lifetimes $\tau$ extracted from the exponential fits shown in the top panel. Times in this plot are measured in units of $2 R / U$ with $R$ the pipe radius and $U$ the mean downstream velocity.

its basin of attraction naturally suggests to look for the boundary between the two objects. In the case of coexisting attractors, this would be the basin boundary. However, in order to cover the case of a transient dynamics, the concept of the edge of chaos was introduced [41/42]37/43]: when crossing from the laminar to the turbulent side by increasing the amplitude of the perturbation one notes and smooth increase in lifetimes on the laminar side and a sensitive dependence on initial conditions on the turbulent side [35]. Inbetween is a first point where the lifetime becomes infinite. Because of the chaotic variations on the turbulent side, this point sits on the edge of chaos. The location of this edge of chaos in state space can be expected to be fairly complex: folds have been identified [37] and fractal properties cannot be ruled out [4445]43. Interestingly, different points on the edge of chaos can be dynamically connected, and it makes sense to probe for the dynamics on the edge of chaos. The evolution of the system then shows that there is a relative attractor: it is attracting for initial conditions in the edge, but globally unstable because of the tendency to either swing up to the turbulent dynamics or to return to the laminar one. We call this relative attractor the edge state, as it is embedded in the edge of chaos. As mentioned before, it extends the concept of basin boundary for attractors to the situation with an attractor and a saddle, see 43 for further discussion. The boundary and its dynamics is also relevant for determining the smallest perturbation sufficient to trigger turbulence, e.g. [46,

On the practical side, the boundary can be tracked by straddling it with a pair of trajectories where one re-

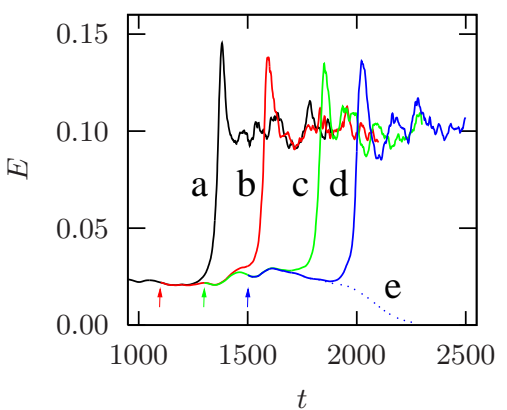

Fig. 8. Visualization of the edge state tracking algorithm for pipe flow. The edge state is bracketed by a pair of initial conditions in which one initial condition decays and the other becomes fully turbulent. After about 200 time units the approximation is refined by choosing a new pair of approximating trajectories. The temporal modulations in energy indicate that the edge state maintains some dynamics and does not become a travelling wave.
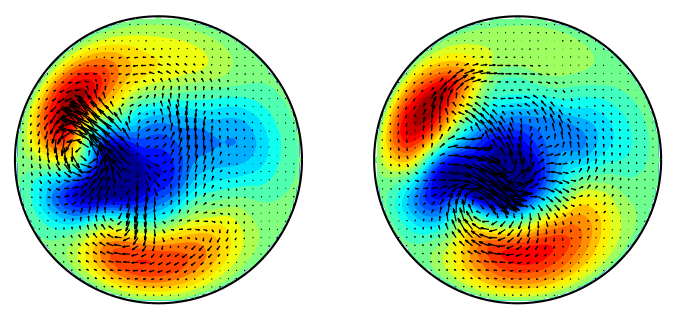

Fig. 9. Two snapshots of the edge state at $R e=2160$. The snapshots clearly show the oscillating motion of the center vortices between the two high-speed regions near the walls.

turns to the laminar state directly, and the other swings up to the turbulent state. Since the dynamics in the edge is unstable, the two trajectories will separate. If the separation becomes large enough, a new pair of trajectories can be found by refinement, see Fig. 8, This way the pair will always stay close to the edge and will approximate a trajectory the never leaves it. The velocity fields for these trajectories become progressively simpler as time goes on [42 37. In the end, a state consisting of two high speed streaks, a low speed streak in the middle, and a pair of vortices that drives the streaks, is obtained, see Fig. 9. Interestingly, the two vortices in the middle are dynamically active, but do not leave this region. This type of state can be followed up to higher Reynolds numbers.

\section{Conclusions}

The studies on the transition to turbulence in pipe flow have confirmed a few expectations (like a subcritical transition scenario), but have also revealed unexpected properties: the lack of stability of the coherent structures, and their transient appearance in turbulent flows, the nonpersistent nature of the turbulent state, the complicated boundary between laminar and turbulent, etc. The effort invested into understanding this transition can also 
be expected to be helpful in other situations: plane Couette flow is perhaps the next flow that comes to mind, as it shares with pipe flow the linear stability of the laminar profile to infinite Reynolds numbers. Pressure driven plane Poiseuille flow does have a linear instability, but at a Reynolds number above the one where turbulence appears. All these flows share many similarities, and can be profitably studied with the concepts presented here.

Financial support by the Deutsche Forschungsgemeinschaft is gratefully acknowledged.

\section{References}

1. E. L. Koschmieder. Bénard Cells and Taylor Vortices. Cambridge University Press, 1993.

2. L. Boberg and U. Brosa. Z. Naturforsch., 43a:697-726, 1988.

3. S. Grossmann. Rev. Mod. Phys., 72:603-618, 2000.

4. R. R. Kerswell. Nonlinearity, 18:R17-R44, 2005.

5. B. Eckhardt, T.M. Schneider, B. Hof, and J. Westerweel. Ann. Rev. Fluid Mech., 39:447-468, 2007.

6. B. Eckhardt, H. Faisst, A. Schmiegel, and J. Schumacher. In I. P. Castro, P. E. Hanock, and T. G. Thomas, editors, Advances in Turbulence IX, pages 701-708, Barcelona, 2002.

7. B. Eckhardt, H. Faisst, A. Schmiegel, and T. M. Schneider. Phil. Trans. R. Soc. (London), in press, 2007.

8. T. Mullin and R. Kerswell (eds). Springer, Dordrecht, 2004.

9. O. Reynolds. Phil. Trans. R. Soc. (London), 174:935-982 +3 plates, 1883 .

10. O. Reynolds. Proc. R. Soc. (London), 35:84-99, 1883.

11. L. Prandtl and O. G. Tietjens. Applied Hydro- and Aeromechanics. Dover Publications, New York, 1934.

12. A. G. Darbyshire and T. Mullin. J. Fluid Mech., 289:83114, 1995.

13. I. J. Wygnanski and F. H. Champagne. J. Fluid Mech., 59:281-335, 1973.

14. I. J. Wygnanski, M. Sokolov, and D. Friedman. J. Fluid Mech., 69:283-304, 1975.

15. M. C. Cross and P. C. Hohenberg. Rev. Mod. Phys., 65:851-1112, 1993.

16. J. Schumacher and B. Eckhardt. Phys. Rev. E, 63:046307, 2001.

17. A. Prigent, G. Gregoire, H. Chaté, O. Dauchot, and W. van Saarloos. Phys. Rev. Lett., 89:014501, 2002.

18. D. Barkley and L. S. Tuckerman. Phys. Rev. Lett., 94:014502, 2005.

19. T. Mullin and J. Peixinho. In T. Mullin and R. Govindarajan, Eds, IUTAM Symposium transition in shear flows. Springer, 2005.

20. T. Mullin and J. Peixinho. J. Low Temp. Phys., 145:75-88, 2006.

21. J. Peixinho and T. Mullin. Phys. Rev. Lett., 96:094501, 2006.

22. J. Peixinho and T. Mullin. J. Fluid Mech., 582:169-178, 2007.

23. B. Hof. In Laminar-turbulent transition and finite amplitude solutions, pages 221-231. Springer, 2004.
24. A.P. Willis and R.R. Kerswell. arxiv, 0706.3330:4 pages, 2007.

25. B. Hof, et al. Science, 305:1594, 2004.

26. T. M. Schneider, B. Eckhardt, and J. Vollmer. Phys. Rev. E, 75:066313, 2007.

27. C. W. H. van Doorne. Stereoscopiv PIV on transition in pipe flow. PhD thesis, TU Delft, 2004.

28. H. Faisst and B. Eckhardt. Phys. Rev. Lett., 91:224502, 2003.

29. E. Ott. Chaos in Dynamical Systems. Cambridge University Press, 1993.

30. H. Faisst and B. Eckhardt. Phys. Rev. E, 61:7227-7230, 2000.

31. H. Wedin and R. R. Kerswell. J. Fluid Mech., 508:333-371, 2004.

32. C. Pringle and R.R. Kerswell. Phys. Rev. Lett., in press, 2007.

33. A. Meseguer and L.N. Trefethen. J. Comp. Phys., 186:178197, 2003.

34. U. Brosa. J. Stat. Phys., 55:1303-1312, 1989.

35. H. Faisst and B. Eckhardt. J. Fluid Mech., 504:343-352, 2004.

36. B. Hof, J. Westerweel, T.M. Schneider, and B. Eckhardt. Nature, 443:60-64, 2006.

37. T. M. Schneider, B. Eckhardt, and J.A. Yorke. Phys. Rev. Lett., 99:034502, 2007.

38. A. P. Willis and R.R. Kerswell. Phys. Rev. Lett., 98:014501, 2007.

39. B. Hof, J. Westerweel, T. M. Schneider, and B. Eckhardt. arXiv:0707.2642v1 [physics.flu-dyn], 2007.

40. G. Ben-Dov and J. Cohen. Phys. Rev. Lett., 98:064503, 2007.

41. J. D. Skufca, J. A. Yorke, and B. Eckhardt. Phys. Rev. Lett., 96:174101, 2006.

42. T. M. Schneider and B. Eckhardt. Chaos, 16:020604, 2006.

43. J. Vollmer, T. M. Schneider, and B. Eckhardt. in preparation, 2007.

44. A. Schmiegel and B. Eckhardt. Phys. Rev. Lett, 79:52505253, 1997.

45. J. Moehlis, H. Faisst, and B. Eckhardt. New J. Phys., 6:56, 2004.

46. F. Mellibovsky and A. Meseguer, Phys. Fluids 18:074104, 2006; ibid, 19:044102, 2007. 